\title{
Hepatitis B virus vaccination failure in celiac disease and type 1 diabetes: what is the truth?
}

\author{
Raffaella Mormile $^{1}$
}

Accepted: 1 September 2015 / Published online: 7 September 2015

(C) Springer-Verlag Berlin Heidelberg 2015

\section{Dear Editor:}

Hepatitis B virus (HBV) is a major public health issue. One third of the world's population has been infected with HBV. Infection with $\mathrm{HBV}$ is an established cause of acute and chronic hepatitis, ranging from fulminant hepatitis to cirrhosis and eventually hepatocellular carcinoma. Vaccination represents the main method of containment. Current recombinant HBV vaccine show excellent immunogenicity. However, approximately 5$10 \%$ of vaccinated individuals fail to mount an adequate antibody response remaining vulnerable to $\mathrm{HBV}$ infection. It has been proposed that unresponsiveness to HBV vaccination may be connected with the presence of specific human leukocyte antigen haplotypes and specific single nucleotide polymorphism (SNPs) in genes of cytokine/cytokine receptors. However, the pathogenic mechanism responsible for the defective production of protective levels of antibodies to HBV vaccine still remains unclear. T helper 1 (Th1)-type cell-mediated response has been shown to be a prerequisite of HBV clearance. Abnormalities in Th1-specific immune responses have been accounted for nonresponsiveness to $\mathrm{HBV}$ vaccine. Among Th1 cytokines, increasing evidence has emerged regarding the significant role of interferon- $\gamma$ (IFN- $\gamma)$ in the protective immunity against HBV. Mutations that interfere with IFN- $\gamma$ production have been linked to differences in clinical presentation and outcome of HBV including the variability of viremia. Development of immunity after $\mathrm{HB}$ vaccination has been correlated with IFN- $\gamma$ gene polymorphism. Intriguingly, genetic variants in the IFN- $\gamma$ gene have

Raffaella Mormile

raffaellamormile@alice.it

1 Division of Pediatrics and Neonatology, Moscati Hospital, Via A. Gramsci, 3, 81031 Aversa, Italy been described to have an impact on the magnitude of immune response to HBV vaccination. Non-responsiveness to HBV vaccine series is reported to occur in both celiac disease (CD) and type 1 diabetes (T1D). CD is an autoimmune enteropathy caused by permanent intolerance to dietary gluten in genetically susceptible individuals resulting in villous atrophy, inflammation, and crypt cell hyperplasia in the proximal intestine. Similarly to HBV vaccine non-response, $\mathrm{CD}$ has been related to a Th1-like cytokine production profile with particular involvement of IFN- $\gamma$ that is considered a key effector in this disorder. It has been reported that IFN- $\gamma$ is the predominant cytokine secreted by glutenreactive $\mathrm{T}$ cells in the celiac intestine. It has been suggested that functional variants of the IFN- $\gamma$ gene have a role in determining CD susceptibility. T1D is a chronic autoimmune disease characterized by the destruction of pancreatic $\beta$ cells leading to absolute insulin deficiency. A Th1 response pattern has also been found to play a central role in T1D development. IFN- is conventionally described as responsible for driving the destruction of pancreatic $\beta$ islets in T1D. Concordantly, it has been observed that IFNreceptor deficiency prevents diabetes. Similarly to unresponsiveness to $\mathrm{HBV}$ vaccination and $\mathrm{CD}$, the IFN- gene polymorphism has long demonstrated to be also associated with T1D onset. All these contentions led us to hypothesize that a particular combination of functional IFN- SNPs and their haplotypes may be associated with the co-occurrence of non-responsiveness to the $\mathrm{HBV}$ vaccine, $\mathrm{CD}$, and T1D in the same individual. Moreover, we suppose the existence of a strong linkage disequilibrium in the functional IFN- SNPs. Because HB vaccination is recommended for all infants, children should be tested for immunity to $\mathrm{HB}$ after completion of the vaccine series in order to select subjects on the basis of their increased risk for CD and T1D. Research studies are needed to identify SNPs in the IFN- $\gamma$ gene to utilize as biomarkers of latent CD and T1D in children with absence of seroconversion following the complete $\mathrm{HB}$ vaccine regimen. 